\title{
Giant-Magnetic Clusters and Susceptibility Enhancement in Pd-Ni Alloys
}

\author{
Nicholas Kioussis \\ Department of Physics, California State University Northridge, Northridge, California 91330 \\ James W. Garland \\ Department of Physics, University of Illinois at Chicago, Chicago, Illinois 60680 \\ (Received 4 April 1991)
}

\begin{abstract}
A recently developed formalism is used to calculate the saturation magnetization and temperaturedependent, exchange-enhanced Pauli susceptibility of paramagnetic and weakly ferromagnetic Pd-Ni alloys. The effects of quantum and thermal spin fluctuations are included in alloy calculations for the first time, through the use of a semiphenomenological theory consistent with the results of renormalizationgroup theory for the single-impurity problem. Two different cluster methods are used, both of which yield results superior to those of all previous theories and in good agreement with experiment.

PACS numbers: $75.20 . \mathrm{En}, 71.70 . \mathrm{Gm}, 75.30 . \mathrm{Cr}, 75.30 . \mathrm{Hx}$
\end{abstract}

Nickel does not form a stable magnetic moment as an isolated impurity atom in nonmagnetic transition-metal hosts, even in a strongly exchange-enhanced host such as Pd [1-3]. However, stable magnetic moments do form in concentrated paramagnetic alloys, in which magnetic clusters are nucleated at $\mathrm{Ni}$ atoms with extremely $\mathrm{Ni}$-rich local environments [4]. Thus, these alloy systems are characterized by an inhomogenous distribution of magnetic polarization clouds, extending over about 200 atoms and having giant magnetic moments [4,5]. Furthermore, the relatively temperature-independent exchangeenhanced Pauli component of the susceptibility exhibits a broad finite peak near the critical concentration for the onset of ferromagnetism [1-3]. Among these Ni-based alloy systems, the $\mathrm{Pd}_{1-} \mathrm{Ni}_{c}$ system has [1-3] the lowest critical concentration $(\approx 2.35$ at. $\% \mathrm{Ni})$, due to the highly polarizable, exchange-enhanced $\mathrm{Pd}$ host, and thus one would expect the calculation to be the least difficult for this system. Magnetization and susceptibility measurements [1-3] have shown that the magnetic clusters in $\mathrm{Pd}-\mathrm{Ni}$ alloys are nucleated by groups of three or more nearest-neighbor $\mathrm{Ni}$ atoms, and also by nearest-neighbor $\mathrm{Ni}$ pairs that have other $\mathrm{Ni}$ pairs and/or other $\mathrm{Ni}$ singles in close proximity, with average cluster moments of about $17 \mu_{B}$ and $12 \mu_{B}$, respectively.

Previous theories of the magnetization and exchangeenhanced susceptibility of concentrated alloys based [6-9] on the single-site coherent-potential approximation (CPA), by the very nature of single-site averaging, fail to incorporate local-environment effects, which are very important in this class of alloys. Previous cluster CPA theories [10], which represent approximations to the present formalism, fail to include exchange-enhancement in the CPA effective medium or to treat correctly atoms or clusters with a large local susceptibility, and fail to predict local moment formation on $\mathrm{Ni}$ atoms even qualitatively. The theory of Kato and Mathon [11] is the only previous theory of the susceptibility of concentrated alloys which gives a nondivergent Pauli susceptibility at all concentrations. However, this theory gives spurious discontinuities in the susceptibility as a function of concentration which can be corrected only by abandoning self-consistency and introducing extra parameters. On the other hand, it is not feasible to perform $a b$ initio cluster band calculations [12] to determine the moments as a function of local environment for the many thousands of local atomic configurations which must be considered for Ni-based alloys. Thus, for the applications intended the theory presented here is uniquely useful.

Here we report calculations of the saturation magnetization and temperature-dependent exchange-enhanced Pauli susceptibility $\chi_{P}(c, T)$ of paramagnetic and weakly ferromagnetic Pd-Ni alloys. We start from our recently developed [13,14] theory of concentrated substitutionally disordered alloys within the random-phase approximation (RPA). However, we must go beyond that theory because spin-fluctuation (SF) effects are neglected within the RPA, yielding a divergence in the local susceptibility on all sites when the local exchange enhancement reaches a critical value on any site. This neglect of SF leads to an overestimate of the susceptibility at all concentrations for any alloy system. For alloy systems which possess a critical concentration for ferromagnetism, the overestimate is serious near that concentration. Moreover, the temperature dependence of the susceptibility is not treated within this theory. We modify our previous RPA results semiphenomenologically so as to include the effects of quantum and thermal SF in a manner consistent with scaling laws and with the results [15] of renormalization-group theory for the single-impurity problem. The temperature-dependent uniform susceptibility associated with any cluster embedded in a pure host or in an effective medium is expressed in terms of the local SF temperature $\theta_{\mathrm{SF}}$ of the cluster, which in turn is expressed in terms of the zero-temperature RPA Pauli susceptibility of the cluster calculated from our previous theory $[13,14]$. This is the first time SF effects have been taken into account in alloy calculations away from the dilute 
limit. The principal physical approximation made in our calculation of $\chi_{P}(c, T)$ is our treatment of each magnetic cluster as a structureless entity, thus neglecting internal SF.

Two different cluster methods are used to calculate $\chi_{P}(c, T)$. In the first method, $\chi_{P}(c, T)-\chi_{P}(0, T)$ is expressed as a sum of incremental contributions $\Delta \chi_{P}^{(n)}(c, T)$ from sets of $n \mathrm{Ni}$ atoms embedded in pure $\mathrm{Pd}$, with $\Delta \chi_{P}^{(n)}(c, T)$ defined so that it vanishes if any subset of the $\mathrm{Ni}$ atoms is moved far away from all of the remaining $\mathrm{Ni}$ atoms. Because of the need to consider very spread-out clusters in this method, we evaluated $\chi_{P}(c, T)$ only through order $n=4$. From an expansion of the experimental [2,3] Pauli susceptibility in powers of $c$, it is clear that our expansion up through $n=4$ is valid approximately up to, but not beyond, 1.85 at. $\% \mathrm{Ni}$. In the second method, compact 55-site clusters consisting of real $\mathrm{Ni}$ and Pd atoms are embedded in a self-consistently determined effective medium having a uniform susceptibility $\bar{\chi}(c, T)$, determined as a function of $\mathrm{Ni}$ concentration from the self-consistency condition $\bar{\chi}(c, 0)=\chi_{P}(c, 0)$ [13], where $\chi_{P}(c, 0)$ is the calculated zero-temperature Pauli susceptibility. In this method we considered up through six $\mathrm{Ni}$ atoms in a cluster, an expansion valid at least up to 3.0 at. $\% \mathrm{Ni}$. Thus, this method permits a comparison with experiment [1-3] and with earlier theories [6-11] through the critical concentration and into the weakly ferromagnetic regime.

For the class of alloy systems considered here, the calculated $\chi_{P}(c, T)$ cannot be compared directly to the experimentally measured $\chi_{\exp }(c, T)$, because the latter contains a substantial Curie-Weiss (CW) component arising from intercluster exchange interactions, which are neglected in this and all previous calculations. Magnetization and susceptibility measurements [1,3] on paramagnetic and weakly ferromagnetic $\mathrm{Pd}-\mathrm{Ni}$ alloys have shown that the low-temperature $\chi_{\exp }(c, T)$ can be resolved into the sum of two components-a CW-like component, $\chi_{\mathrm{CW}}(c, T)=C_{\mathrm{CW}}(c) /\left(T+\Theta_{\mathrm{CW}}\right)$, which arises from magnetic clusters, and a relatively temperature-independent Pauli component $\chi^{\prime}(c, T)$. In these alloys $\chi^{\prime}(c, T)$ $\neq \chi_{P}(c, T)$, because $\chi_{P}(c, T)$ also contains a substantial $C W$-like component arising from the large $S F$ associated with clusters which within the RPA almost form or just barely form magnetic moments. For any given cluster these SF give rise to a susceptibility which differs by less than $1 \%$ from the $\mathrm{CW}$ form for $T \geq 2 T_{K}$, with the $\mathrm{CW}$ temperature of the cluster equal to $4 T_{K} / 3$, where $T_{K}$ is the Kondo temperature [15] of that cluster. Therefore, in order to compare the calculated $\chi_{P}(c, T)$ with experiment, we assume the experimentally determined values of $\Theta_{\mathrm{CW}}$ to be the true $\mathrm{CW}$ temperatures, so that the calculated $\chi^{\prime}(c, T)$ can be determined [16] by fitting the calculated $\chi_{P}(c, T)$ with the form $\chi_{P}(c, T)=\chi^{\prime}(c, T)+C^{\prime}(c) /$ $\left(T+\Theta_{\mathrm{CW}}\right)$ at low temperatures. Here, $\chi^{\prime}(c, T)$ is assumed to be temperature independent for $T \leq 10 \mathrm{~K}$, and
$C^{\prime}(c)$ is the $\mathrm{CW}$-like component arising from barely magnetic or highly exchange-enhanced Ni clusters. The experimental $C(c)$ is to be identified as the sum of $C^{\prime}(c)$ and the $\mathrm{CW}$ constant, $C_{\mathrm{CW}}(c)$ associated with stable moments. The calculated values [16] of $C(c)$ are found to be in excellent agreement with experiment.

The enhancement of the local susceptibility in the vicinity of $\mathrm{Ni}$ atoms in $\mathrm{Pd}-\mathrm{Ni}$ alloys arises [13,14] (1) from an enhancement of the local band susceptibilities, $\Gamma_{i j}$ $\equiv \partial \mu_{i} / \partial H_{j}$, if either or both of the sites $i$ and $j$ are $\mathrm{Ni}$ sites, and (2) from the presumably larger value of the intra-atomic Coulomb interaction $U$ on $\mathrm{Ni}$ sites $\left(U_{\mathrm{Ni}}\right.$ $\left.=U_{\mathrm{Pd}}+\delta U\right)$. Calculations were performed for three cases, with a single adjustable parameter in each case: (a) diagonal disorder (only $\delta U \neq 0$ ); (b) off-diagonal disorder only (only $\delta U=0$ ); and (c) off-diagonal disorder with $\eta^{2} \equiv \Gamma_{i j}^{\mathrm{Ni}-\mathrm{Pd}} / \Gamma_{i j}^{\mathrm{Pd}-\mathrm{Pd}}$ fixed at the value of 1.85 , found [14] in model band-structure calculations (with $\delta U$ adjustable) for clusters of one or two $\mathrm{Ni}$ impurities. Because the results are very similar for all three cases, we report here only results for the case of diagonal disorder and with $\delta U=1.276 \mathrm{eV}$, which yields [16] the best agreement with experiment for $\chi^{\prime}(c, 0)$ using the first cluster method.

We found $\Theta_{\mathrm{SF}}=2.45 T_{K}$ to be $450 \mathrm{~K}$ for an isolated $\mathrm{Ni}$ impurity in pure $\mathrm{Pd}, 130 \mathrm{~K}$ for a nearest-neighbor $\mathrm{Ni}$ pair, and $300 \mathrm{~K}$ for a second-nearest-neighbor $\mathrm{Ni}$ pair. Thus, isolated $\mathrm{Ni}$ impurities and $\mathrm{Ni}$ pairs contribute almost solely to $\chi^{\prime}(c, 0)$ and hardly at all to the $\mathrm{CW}$-like term in $\chi_{P}(c, T)$. All $\mathrm{Ni}$ triplets in which one $\mathrm{Ni}$ atom was a nearest-neighbor to both of the others were found to form local moments within the RPA. However, almost all other isolated triplet configurations were found to have values of $\Theta_{\mathrm{SF}} \geq 100 \mathrm{~K}$ and, hence, to contribute primarily to $\chi^{\prime}$. Values of $\Theta_{\mathrm{SF}}$ for nonmagnetic $\mathrm{Ni}$ quartets in pure $\mathrm{Pd}$ at 1.5 at. $\% \mathrm{Ni}$ are shown in Fig. 1. Although most of

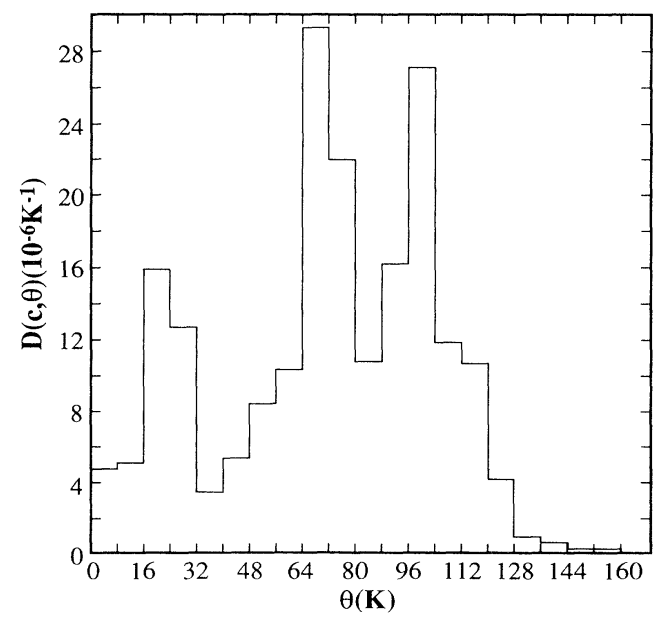

FIG. 1. Distribution of the calculated spin-fluctuation temperatures for $\mathrm{Ni}$ quartets embedded in pure Pd for 1.5 at. $\% \mathrm{Ni}$. 


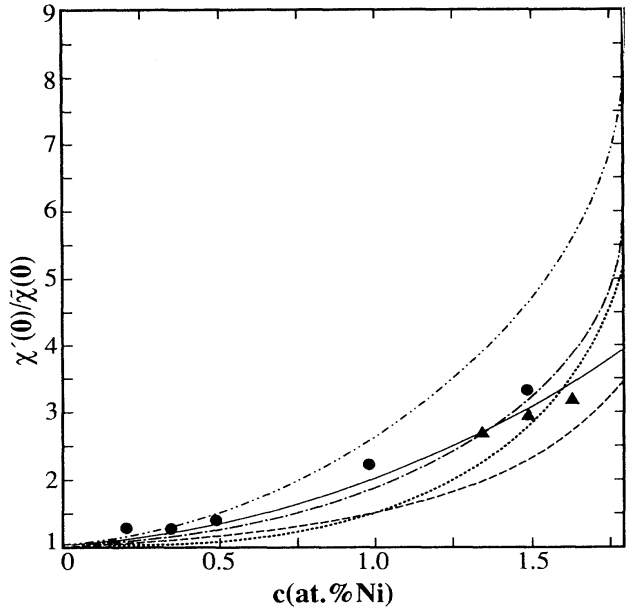

FIG. 2. Results for $\chi^{\prime}(c, 0)$ obtained using the first method (solid curve), normalized to the susceptibility of pure Pd, compared with those of previous calculations, Ref. [6] (---), Ref.

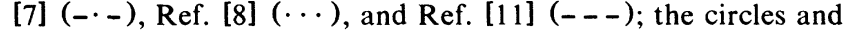
triangles are the experimental results from Refs. [1] and [2], respectively.

the nonmagnetic $\mathrm{Ni}$ quartets have $\Theta_{\mathrm{SF}}$ between 48 and $120 \mathrm{~K}$, and hence contribute primarily to $\chi^{\prime}$, a substantial fraction of them have $\Theta_{\mathrm{SF}} \leq 32 \mathrm{~K}$. Thus, for low Ni concentration we find $\mathrm{Ni}$ quartets to be the dominant source of the CW-like term in $\chi_{P}(c, T)$.

The results obtained for $\chi^{\prime}(c, 0)$ using the first cluster method, normalized to the susceptibility of pure $\mathrm{Pd}$, are shown in Fig. 2 ( solid curve) and compared with the results of previous calculations $[6-8,11]$ and with experiment $[1,2]$. Our results are in far better agreement with experiment than are those of previous calculations over that concentration range. The corresponding results for $\chi^{\prime}(c, 0)$ obtained using the second method are shown in Fig. 3 ( solid curve) along with the results of previous calculations [7,11] and experiment [1-3]. These results were obtained using the value of $\delta U$ which gave the best results at lower Ni concentrations using our first method, and thus in a sense are zero-parameter results. As can be seen, this value of $\delta U$ gives results which are systematically too high using our second method. However, our results show a broad peak in $\chi^{\prime}$ with very nearly the same width as the experimental results, unlike the results of previous calculations. Furthermore, our results were obtained without abandoning self-consistency or introducing extra parameters, as was done by Kato and Mathon [11]. The reduction of $\chi^{\prime}$ at higher $\mathrm{Ni}$ concentrations $(c \geq 2.5$ at. $\% \mathrm{Ni}$ ) is due to those $\mathrm{Ni}$ quintets and sextets which are associated with stable magnetic moments. The systematic overestimate of $\chi^{\prime}$ using the second method results from the extremely nonlinear dependence of the susceptibility of an atom on its local environment, coupled with the extremely long range of the susceptibility in Pd$\mathrm{Ni}$ alloys. The embedding of clusters in an effective

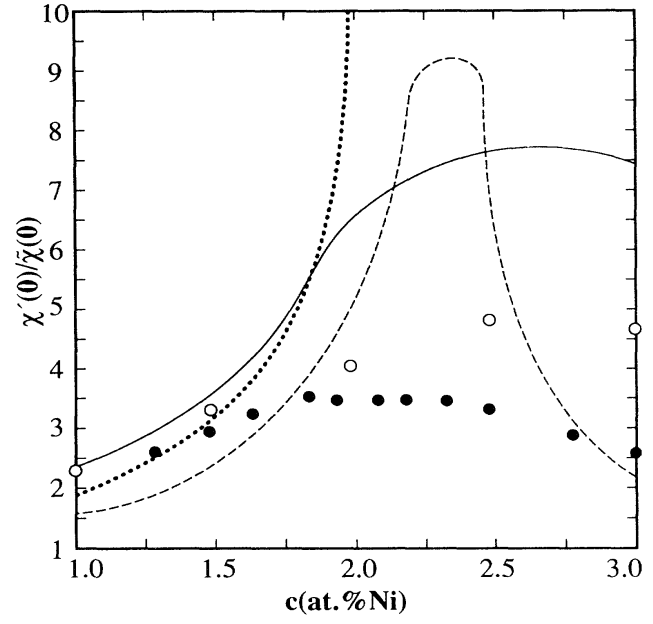

FIG. 3. Results for $\chi^{\prime}(c, 0)$ obtained using the second method (solid curve), normalized to the susceptibility of pure Pd, compared with those of previous calculations, Ref. [7] ( . . ) and Ref. [11] (---); the open and solid circles are the experimental results from Refs. [1] and [3], respectively.

medium replaces that nonlinear dependence by a linear one, thus producing a systematic overestimate of $\chi^{\prime}$, especially in the vicinity of the critical concentration. This overestimate is greatly amplified by positive feedback in establishing self-consistency [16], and by the large number of cluster configurations in which one or more $\mathrm{Ni}$ atoms are very close to the cluster edge.

In calculating the average cluster moment $\mu^{*}(c)$, the saturation magnetization $M(c)$, and the concentration $c^{*}(c)$ of magnetic clusters, we used [14] a cutoff radius of $9.0 \AA$ for the magnetic polarization clouds. This value was determined from percolation theory to fit the experi-

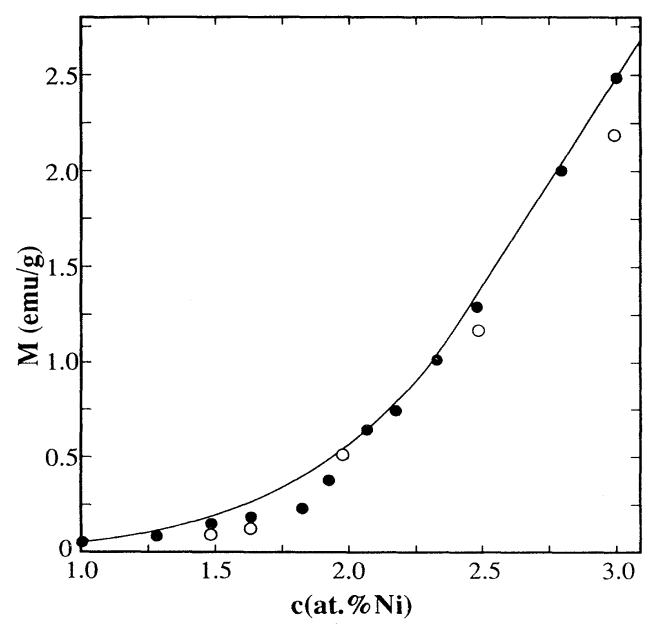

FIG. 4. Calculated saturation magnetization $M$; the open and solid circles are the experimental results from Refs. [1] and [3], respectively. 


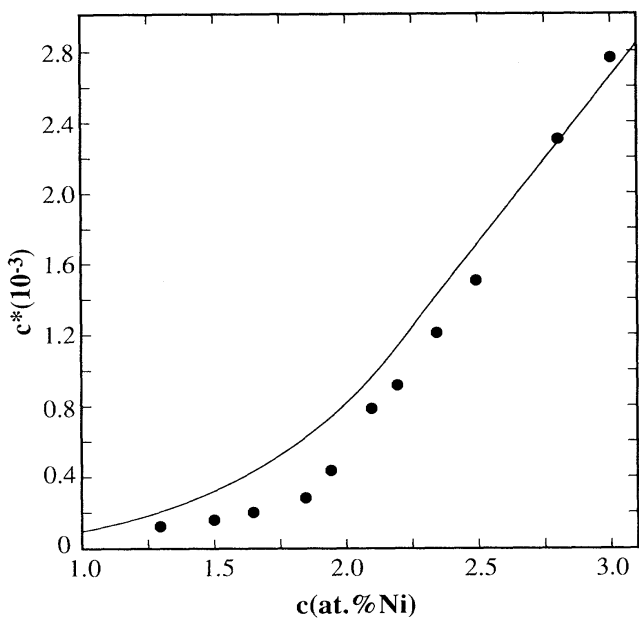

FIG. 5. Calculated concentration $c^{*}$ of magnetic polarization clouds; the circles are the experimental results from Ref. [3].

mentally observed critical concentration. We find $\mu^{*}$ to increase linearly with $c$ from about $7 \mu_{B}$ at 1 at. $\% \mathrm{Ni}$ to about $18 \mu_{B}$ at 3.0 at. $\% \mathrm{Ni}$, a result intermediate between the experimental results of Cheung, Kouvel, and Garland [3], who found $\mu^{*}$ to be constant at a value of $15 \mu_{B}$, and those of Chouteau [1], who found $\mu^{*}$ to increase from $10 \mu_{B}$ to $16 \mu_{B}$ from 1.3 to 1.65 at. $\% \mathrm{Ni}$. The calculated values for $M$ and $c^{*}$ are compared with the experimental results $[1,3]$ in Figs. 4 and 5, respectively. The theoretical values of $M$ and $c^{*}$ are in very good agreement with experiment for $c>2$ at. \% $\mathrm{Ni}$, but are higher than the experimental values in the lower concentration range. However, in that concentration range one expects the experimental values to be reduced by the existence [17] of a spin-glass state. In comparison, the only other calculations [11] yield the unphysical result of a vanishing saturation magnetization above approximately 2 at.\% $\mathrm{Ni}$. The majority of magnetic clusters have cluster moments in the range $(6-12) \mu_{B}$ at 1.0 at. $\% \mathrm{Ni},(12-18) \mu_{B}$ at 2.0 at. $\% \mathrm{Ni}$, and $(18-24) \mu_{B}$ at 3.0 at. $\% \mathrm{Ni}$.

One of us (N.K.) acknowledges support by the School of Science and Mathematics and the Office of Research and Sponsored Projects at California State University Northridge.

[1] G. Chouteau, Physica (Amsterdam) 84B, 25 (1976).

[2] D. Sain and J. S. Kouvel, Phys. Rev. B 17, 2257 (1978).

[3] T. D. Cheung, J. S. Kouvel, and J. W. Garland, Phys. Rev. B 23, 1245 (1981).

[4] A. T. Alfred, B. D. Rainford, and M. W. Stringfellow, Phys. Rev. Lett. 24, 897 (1970); J. W. Cable and H. R. Child, Phys. Rev. B 1, 3809 (1970).

[5] J. W. Garland and N. Kioussis, J. Appl. Phys. 55, 2356 (1984).

[6] R. Harris and M. J. Zuckermann, Phys. Rev. B 5, 101 (1972).

[7] T. Kato and M. Shimizu, J. Phys. Soc. Jpn. 33, 363 (1972).

[8] K. Levin, R. Bass, and K. H. Bennemann, Phys. Rev. B 6, 1865 (1972).

[9] H. Hasegawa and J. Kanamori, J. Phys. Soc. Jpn. 31, 382 (1971).

[10] F. Brouers, F. Gautier, and J. van der Rest, J. Phys. F 5, 975 (1975); J. van der Rest, ibid. 7, 1051 (1977).

[11] T. Kato and J. Mathon, J. Phys. F 6, 221 (1976); 6, 1341 (1976).

[12] A. Rodriguez and J. Keller, J. Phys. F 11, 1423 (1981); B. Delley, D. E. Ellis, and A. J. Freeman, J. Magn. Magn. Mater. 30, 71 (1982).

[13] N. Kioussis and J. W. Garland, Phys. Rev. B 37, 3611 (1988).

[14] N. Kioussis and J. W. Garland, Phys. Rev. B 37, 3626 (1988).

[15] H. R. Krishnamurthy, K. G. Wilson, and J. W. Wilkins, Phys. Rev. Lett. 35, 1101 (1975).

[16] N. Kioussis, J. W. Garland, and J. S. Kouvel (to be published).

[17] T. D. Cheung and J. S. Kouvel, Phys. Rev. B 28, 3831 (1983). 